\title{
Lateral oxidation of InAIAs in InP-based heterostructures for long wavelength vertical cavity surface emitting laser applications
}

\author{
H. Gebretsadik, K. Kamath, W-D. Zhou, and P. Bhattacharyaa) \\ Department of Electrical Engineering and Computer Science, Solid State Electronics Laboratory, University \\ of Michigan, Ann Arbor, Michigan 48109-2122 \\ C. Caneau and R. Bhat \\ Bellcore, Redbank, New Jersey 01701-5699
}

(Received 22 September 1997; accepted for publication 7 November 1997)

\begin{abstract}
We have studied the wet thermal oxidation of $\operatorname{In}_{0.52} \mathrm{Al}_{0.48} \mathrm{As}$ and its potential application in current and optical confinement in vertical cavity surface emitting lasers (VCSELs). Two types of InP-based heterostructures were used to study the effect of adjacent layer compositions on the lateral oxidation behavior of the InAlAs. It was found that the oxidation of $\operatorname{In}_{0.52} \mathrm{Al}_{0.48} \mathrm{As}$ with $\mathrm{InP}$ adjacent layers, compared with $\operatorname{In}_{0.53} \mathrm{Ga}_{0.47} \mathrm{As}$ adjacent layers, proceeded faster, more uniformly and with minimal degradation of the surrounding layers making it ideal for optoelectronic applications. (C) 1998 American Institute of Physics. [S0003-6951(98)00602-0]
\end{abstract}

Recent advances in the wet oxidation of $\mathrm{Al}_{x} \mathrm{Ga}_{1-x} \mathrm{As}$ layers ${ }^{1}$ in GaAs-based heterostructures have led to the dramatic improvements in vertical cavity surface emitting laser (VCSEL) performance. Uses of this native oxide as a dielectric aperature for optical and current confinement have enabled ultra-low threshold currents ${ }^{2-4}$ and high-output power efficiencies exceeding $50 \%{ }^{5}$ in GaAs-based VCSELs. In contrast, advances in the selective oxidation of InAlAs lattice-matched to InP have been slower. This native oxide has been employed in gain-guided long-wavelength InAlAsInP-InGaAsP quantum well laser diodes, ${ }^{6}$ and as a gate insulator in a InAlAs/InGaAs metal-oxide-semiconductor field effect transistor (MOSFET). ${ }^{7}$ Also recently, a highcontrast and high reflectivity Distributed Bragg Reflector (DBR) mirror was obtained using alternating layers of InP and this oxide. ${ }^{8}$

The low $\mathrm{Al}$ content in InAlAs lattice-matched to InP has resulted in very slow lateral oxidation rates as compared to the rate of oxidation of high $\mathrm{Al}$ content AlGaAs. To speed up this process, the oxidation must be performed at very high temperatures, ${ }^{9}$ making it difficult to selectively oxidize laser structures. So far, no study has focused on the selective oxidation of a InAlAs layer in a VCSEL heterostructure with the intent to use it as a dielectric aperture. Much of the current effort is concentrated on characterizing the wet oxidation of AlAsSb lattice-matched to InP, which has an $\mathrm{Al}$ mole fraction of 1.0 and can be performed at lower temperatures. ${ }^{10}$ With the oxidation of AlAsSb, an additional layer of residual elemental $\mathrm{Sb}$ forms above the oxidizing layer, deforming the surrounding layer and possibly posing limitations on its use in optoelectronic devices. We have reported the successful growth of high reflectivity defect-free GaAs/AlAs DBR mirrors on patterned InP-based heterostructures and, have demonstrated the feasibility of monolithic VCSELs utilizing such mirrors through photoluminescence measurements. ${ }^{11}$ Another option is to fabricate a hybrid VCSEL with doublefused AlAs/GaAs mirrors. ${ }^{12}$ In either case, the successful oxidation of InAlAs in a VCSEL structure is a key step to-

${ }^{\text {a)} E l e c t r o n i c ~ m a i l: ~ p k b @ e e c s . u m i c h . e d u ~}$ wards the realization of low-threshold long-wavelength VCSELS.

Two types of $p-i-n$ structures were used in this experiment. The first structure, shown in Fig. 1(a), was grown by

$\longrightarrow$\begin{tabular}{|cl|}
\hline $3000 \AA$ & $p+I n G a A s$ \\
\hline $1200 \AA$ & $p+I n A I A s$ \\
\hline $3000 \AA$ & $i-I n G a A s$ \\
\hline $1000 \AA$ & $n-I n G a A s$ \\
\hline$n+I n P$ Substrate
\end{tabular}

(a)



(b)

FIG. 1. Structures used in this study: (a) InGaAs/InAlAs/InGaAs multilayer and (b) VCSEL heterostructure. (The arrow points to the selectively oxidized layer.) 
molecular beam epitaxy (MBE). It consisted of a $1200 \AA$ thick InAlAs grown between two layers of InGaAs (3000 $)$, lattice-matched to InP. The second structure, grown by metal-organic vapor phase epitaxy (MOVPE), is the active region of a VCSEL structure. It consists of a straincompensated InGaAsP $\lambda$ cavity designed for $1.55 \mu \mathrm{m}$ operation. The structure is schematically shown in Fig. 1(b). The InAlAs layer is $1197 \AA$ thick and is directly above the InP spacer and, below a $\lambda / 4 \mathrm{InP}$ contact layer. This structure was designed $^{13}$ for the fabrication of a hybrid VCSEL utilizing defect-free GaAs/AlAs top DBR mirror and a multilayer dielectric stack bottom mirror.

To prepare the samples for this study, a $3000 \AA$ Alayer of $\mathrm{SiO}_{2}$, acting as a capping layer for the high temperature oxidation, was deposited by plasma-enhanced chemical vapor deposition (PEVCD). Following this deposition, the samples were patterned into $40-105 \mu \mathrm{m}$ stripes. The oxide was dryetched and ridges of dimension $90-110 \mu \mathrm{m}$ were formed using a saturated bromine water (SBW) solution to expose the edges of the InAlAs layer. A section of the VCSEL structure was also patterned into mesas, ranging in diameters of $20-40 \mu \mathrm{m}$ to investigate how the perimeter geometry of the oxidation front affects the oxidation rate. In addition, a section of the InGaAs/InAlAs structure was left uncapped and oxidized for different lengths of times. The samples were all solvent cleaned and placed on InP substrates to avoid damaging the InP substrate, prior to oxidation. ${ }^{7}$ The oxidation was carried out in a horizontal quartz tube in a three-zone furnace fed by nitrogen gas passed through a water bubbler maintained at $95^{\circ} \mathrm{C}$. The nitrogen flow was regulated at 75 $\mathrm{sccm}$. Because the amount of water fed into the furnace is dependent on the water level in the bubbler, it is necessary to start each oxidation run with the same water level.

The lateral oxidation depths of the various ridge samples were measured from cross-sectional scanning electron microscope (SEM) photographs. The depth of lateral oxidation of InAlAs in the VCSEL structure was measured as a function of time and temperature and the data are plotted in Fig. 2(a). The depth of oxide formation as a function of oxidation time is a best fit to a square-root function which is in agreement with already published work on the wet-oxidation of InAlAs. ${ }^{7}$ This confirms that oxidant diffusion is the rate limiting mechanism in this case. It was also observed that the rate of lateral oxidation in the VCSEL structure under identical conditions is faster than that in the InGaAs/InAlAs/ InGaAs structure. This is illustrated in Fig. 2(b). It is our belief that the As rich layers in the InGaAs/InAlAs structure inhibit the desorption of As during the oxidation process. At elevated temperatures, the loss of As from these layers may create an overpressure which may suppress further loss.

The effect of a capping layer on the oxidation process was also investigated. As shown in Fig. 3, the wet-oxidation of InAlAs proceeded faster in the uncapped InGaAs/InAlAs/ InGaAs structure compared to a capped sample but resulted in nonuniform oxidation, a discontinuous oxidation front, and in the degradation of the layers surrounding the InAlAs layers. This nonuniformity in oxidation was not observed in the VCSEL structure, when oxidized without a capping layer. No deformations of the surrounding InP and InGaAsP layers in the VCSEL structure were detected during close

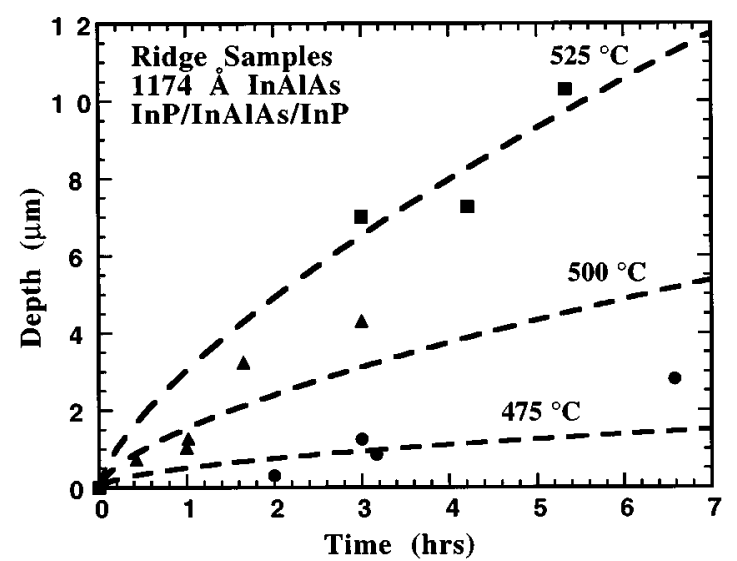

(a)

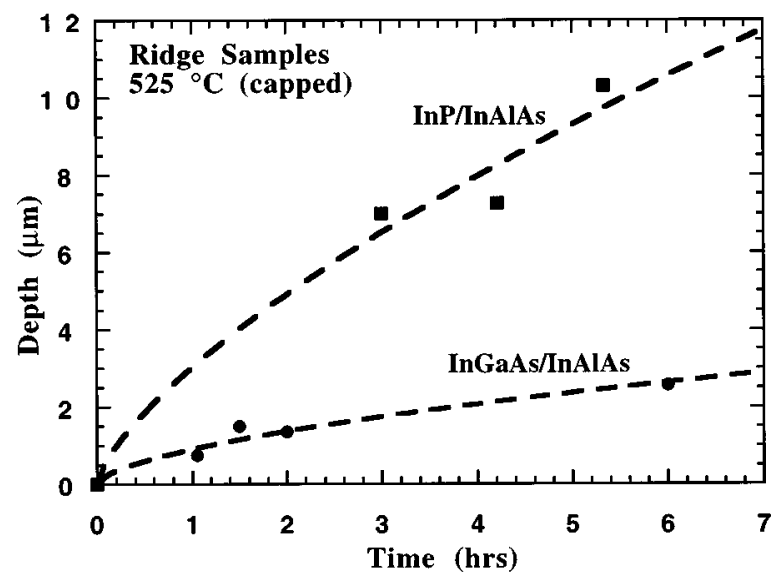

(b)

FIG. 2. Temporal dependence of the lateral oxidation depth of: (a) InAlAs at 475, 500, and $525^{\circ} \mathrm{C}$ and (b) InAlAs in the InGaAs/InAlAs/InGaAs and VCSEL structure at $525^{\circ} \mathrm{C}$.

examination of cleaved samples using a scanning electron microscope, as shown in Fig. 4.

It is important to mention that the surface morphology was affected by the high temperature oxidation. This degradation is not often reported in the literature but, remains a stumbling block in the fabrication of devices utilizing these native oxides. In the GaAs-based devices, a common prob-

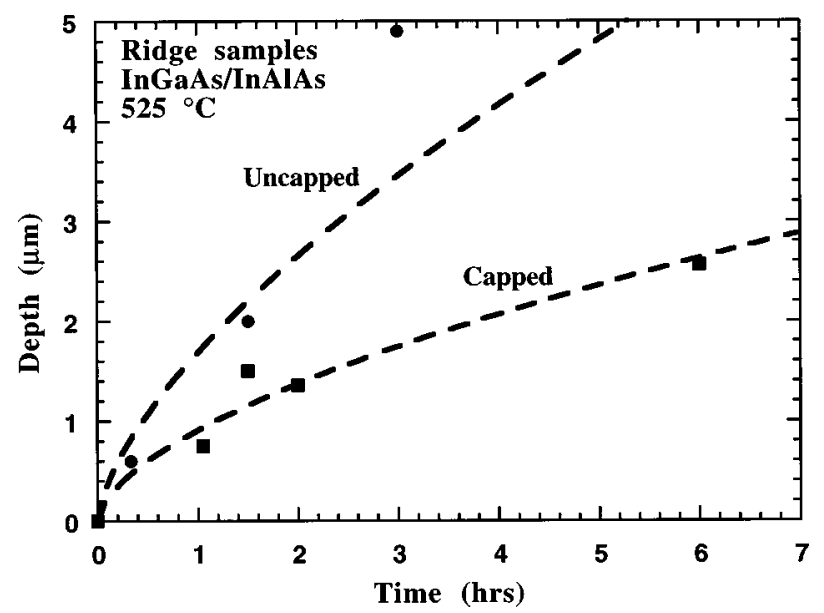

FIG. 3. Temporal dependence of the lateral oxidation depth of InAlAs in the InGaAs/InAlAs/InGaAs with and without the use of a dielectric capping layer. 


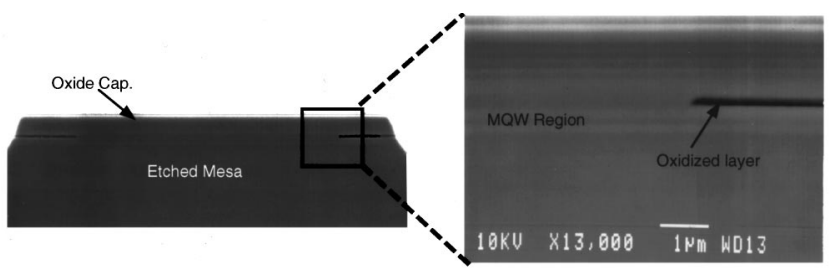

FIG. 4. Scanning electron micrographs of the lateral oxidation of InAlAs in the VCSEL heterostructure, showing the formation of a dielectric current aperture and the oxide terminus.

lem is with delamination of the device resulting from the shrinkage of the oxidizing layer. With InP-based devices, the device samples experience heavy $\mathrm{P}$ loss in the exposed InP regions at high temperatures and become pitted. We found that pitting resulting from this loss was minimal at $475^{\circ} \mathrm{C}$ but otherwise significant over $500{ }^{\circ} \mathrm{C}$ and in cases of overoxidation. Therefore, oxidation of InP-based heterostructures should be performed at temperatures below the congruent evaporation temperature of $\mathrm{InP}$, which is $\sim 480^{\circ} \mathrm{C}$.

In conclusion, the rate of oxidation of InAlAs was found at various temperatures. The oxidation was found to be mainly a diffusive process, with an additional dependence on the As concentration in adjacent layers. The oxidation rate decreased significantly when a dielectric capping layer was used.
The authors wish to thank Dr. X. Zhang for useful discussions and additional growth of samples. This work is being supported by ONR under Grant No. N0014-96-1-0024 and ARO (URI Program) under Grant. No. DAAL03-92-G0109 .

${ }^{1}$ J. M. Dallesasse, N. Holonyak, Jr., A. R. Sugg, T. A. Richard, and N. El-Zein, Appl. Phys. Lett. 57, 2844 (1990).

${ }^{2}$ Y. Hayashi, T. Mukaihara, N. Natori, N. Ohnoki, A. Matsutani, F. Koyama, and K. Iga, Electron. Lett. 31, 550 (1995).

${ }^{3}$ D. L. Huffaker, J. Shin, and D. G. Deppe, Appl. Phys. Lett. 65, 97 (1994).

${ }^{4}$ G. M. Yang, M. H. MacDougal, and P. D. Dapkus, Electron. Lett. 31, 886 (1995).

${ }^{5}$ K. L. Lear, K. D. Choquette, R. P. Schneider, Jr., S. P. Kilcoyne, and K. M. Geib, Electron. Lett. 31, 208 (1995).

${ }^{6}$ S. J. Caracci, M. R. Krames, and N. Holonyak, J. Appl. Phys. 75, 2706 (1994).

${ }^{7}$ P. A. Grudowski, R. V. Chelakara and R. D. Dupuis, Appl. Phys. Lett. 69, 388 (1994).

${ }^{8}$ H. Takenouchi, T. Kagawa, Y. Ohiso, T. Tadokoro, and T. Kurokawa, Electron. Lett. 32, 1671 (1996).

${ }^{9}$ K. Uomi, S. J. Yoo, A. Scherer, R. Bhat, N. C. Andreadakis, C. E. Zah, M. A. Koza, and T. P. Lee, IEEE Photonics Technol. Lett. 6, 317 (1994).

${ }^{10}$ O. Blum, K. M. Geib, M. J. Hafich, J. F. Klem, and C. I. H. Ashby, Appl. Phys. Lett. 68, 3129 (1996).

${ }^{11}$ H. Gebretsadik, K. Kamath, K. K. Linder, X. Zhang, P. Bhattacharya, C. Caneau, and R. Bhat, Appl. Phys. Lett. 71, 581 (1997).

${ }^{12}$ D. I. Babic, J. J. Dudley, K. Streubel, R. P. Mirin, J. E. Bowers, and E. L. Hu, Appl. Phys. Lett. 66, 1030 (1995).

${ }^{13} \mathrm{~V}$. Swaminathan and A. T. Macrander, Materials Aspect of GaAs and InP Based Structures (Prentice Hall, Englewood Cliffs, NJ, 1991), Ch. 2. 